\title{
Influence du stage actif chez le praticien sur le sentiment de compétence professionnelle de l'étudiant en chirurgie dentaire
}

\section{Contribution of private practice training on the self-efficacy belief of French dental surgery students}

\author{
Hélène BAUdin ${ }^{1}$, Emmanuel Nicolas ${ }^{1,2}$, Bernard CHAUMEIL ${ }^{3}$ et Valérie RogER-LERoI ${ }^{1,2}$ \\ 1 Université d'Auvergne, EA 3847, UFR Odontologie, 11 boulevard Charles de Gaulle, 63000 Clermont-Ferrand, France \\ 2 Centre hospitalier régional et universitaire (CHRU) de Clermont-Ferrand, Service d'Odontologie \\ 3 Université d'Auvergne, Unité d'informatique, de pédagogie et des stages (UIPS) en milieu professionnel, Unité de \\ formation et de recherche (UFR) d'odontologie
}

Manuscrit reçu le $1^{\text {er }}$ avril 2009 ; commentaires éditoriaux formulés aux auteurs le 14 août 2009 et le 10 février 2010 ; accepté pour publication le 12 février

\begin{abstract}
Mots clés :
Stage chez le praticien ;

acquisition

des compétences

professionnelles ;

assurance qualité

Résumé - Contexte : Un stage actif d'initiation à la vie professionnelle a été mis en place dans le cursus des étudiants en odontologie au cours de l'année universitaire 2007-2008. À l'UFR de Clermont-Ferrand, les étudiants en dernière année d'études exercent pendant deux mois à temps complet au sein d'un cabinet libéral sous la responsabilité d'un maître de stage. Buts : L'objectif de cette étude est de mettre en évidence l'influence de ce stage sur le sentiment de compétence ou d'efficacité ressenti par les étudiants. Collectifs et méthodes : Une promotion d'étudiants $(n=42)$ a évalué, grâce à une échelle visuelle analogique non graduée, son niveau de performance concernant 52 capacités professionnelles définies par le réseau thématique européen DentEd. Cette auto-évaluation s'est faite à trois reprises : en fin de cinquième année (D3) et au cours de la dernière année d'études (T1) avant puis après le stage actif. Les maîtres de stage qui ont accueilli les étudiants ont à leur tour évalué les capacités des étudiants en utilisant le même procédé. Résultats : En comparant les résultats des trois auto-évaluations par des tests statistiques, notre étude a montré un réel impact positif du stage actif sur le sentiment de compétence perçu par nos étudiants concernant des capacités professionnelles telles que la gestion financière et administrative, la comptabilité, la stérilisation, la gestion des personnels et l'élaboration d'un plan de traitement multidisciplinaire. Pour la plupart des capacités ou habiletés évaluées, il n'existe pas de différence significative entre l'évaluation des étudiants et celle des maitres de stage. Conclusion : Le stage actif donne à l'étudiant le sentiment de mieux maîtriser certaines capacités ou habiletés qui sont difficilement acquises en milieu hospitalier.
\end{abstract}




\section{Keywords:}

Training period; private dental practice; acquiring professional skills; quality assurance
Abstract - Context: A mandatory training period in a private practice was implemented in the curriculum of the French dental students during the 2007-2008 academic year. At the Clermont-Ferrand dental school, final year students practice full-time under the supervision of a dental practitioner for two months. Aim: The aim of this study is to show the potential role of this period on the self-assessed competency perceived by students concerning their professional skills. Methods: Each student of a same cohort $(n=42)$ self-assessed his/her level of performance on a non graduated visual analogue scale. 52 professional skills defined by the European thematic network DentEd were thus assessed. This self-evaluation was performed three times : at the end of the fifth year (D3) and during the final year of studies (T1) before and after the training period. The trainers who supervised the students assessed them using the same scale. Results: When comparing the results of the three self-assessments with statistical tests, our study showed a real positive impact of the training period on the self-efficacy belief concerning professional skills such as financial and administrative management of a private practice, sterilization of material, management of staff and development of a multidisciplinary treatment plan. For most skills, there is no significant difference between the students' and the trainers' assessments. Conclusion: A training period in a private practice allows dental students to feel more competent concerning professional skills which are generally difficult to master in a hospital environment.

\section{Introduction}

Pour exercer la profession de chirurgien-dentiste de manière autonome et indépendante, l'acquisition d'un certain nombre de compétences est indispensable. Celles-ci peuvent se définir comme un système intégré et combinatoire de capacités qui s'appuient elles-mêmes sur un répertoire d'attitudes, de connaissances et d'aptitudes manuelles, nécessaires pour que le jeune diplômé soit capable de résoudre l'ensemble des problèmes de santé orale qu'il rencontrera au cours de son exercice ${ }^{[1]}$. Au cours de ses études, l'étudiant en chirurgie dentaire va acquérir ces compétences notamment en pratiquant des actes courants sur patients, à partir de la troisième année d'études jusqu'à la fin de la sixième année. Cette pratique s'exerce en majeure partie au sein des services hospitaliers d'odontologie appartenant aux Centres hospitaliers régionaux universitaires (CHRU). Or $93 \%$ des étudiants ont ensuite un exercice libéral, différent de la pratique hospitalière, qui a été le modèle de travail de l'étudiant tout au long de ses années d'études. Une immersion au sein d'un cabinet dentaire où l'étudiant pourrait exercer son art en «autonomie contrôlée » pourrait permettre une «transition douce » entre les deux structures. De plus, un stage au sein d'un cabinet libéral peut permettre d'acquérir certaines capacités ou habiletés qu'il est difficile de maitriser dans une structure hospitalière ${ }^{[2]}$. C'est le cas par exemple de l'apprentissage de la stérilisation du matériel, de la gestion des personnels (assistante, réceptionniste) et du laboratoire de prothèse, du tri des déchets, de l'ergonomie du travail, de la gestion du cabinet (comptabilité, obligations légales) et des relations avec les organismes professionnels (Ordre, organismes socio-professionnels, organismes d'assurance maladie...).

Beaucoup de pays européens (Grande-Bretagne, Suisse, Finlande, Pologne, Danemark, ... ) ont déjà introduit dans leur cursus ce type de stage. Il est généralement désigné par la locution «Vocational Training ». En Grande-Bretagne, où de nombreuses études ont été publiées sur le sujet, il est admis que ce stage est bénéfique et il fait maintenant partie intégrante du cursus ${ }^{[3,4]}$. Il est organisé à la fin des études, une fois l'étudiant diplômé et avant qu'il ne soit autorisé à s'inscrire à l'équivalent de notre Ordre professionnel, le General Dental Council $(\mathrm{GDC})^{[5]}$. La durée de ce stage varie selon les pays : elle est d'un an en Grande-Bretagne, en Pologne et au Danemark, de deux ans en Allemagne et de trois ans en République tchèque ${ }^{[2]}$. Comme en France, le jeune diplômé est accueilli dans un 
cabinet libéral, il y exerce en semi-autonomie et est supervisé par un confrère, maître de stage agréé.

Le stage actif d'initiation à la vie professionnelle a fait son apparition dans le cursus des étudiants français en chirurgie dentaire au cours de l'année universitaire 2007-2008. Ses modalités d'application sont définies dans l'arrêté du 24 mai 2005 paru au Journal officiel du 31 mai 2005. Le stage, dont la seule modalité d'organisation précisée dans l'arrêté concerne sa durée minimale de 200 heures, doit permettre à l'étudiant de mettre en application, dans le cadre d'une autonomie contrôlée, les connaissances théoriques, pratiques et cliniques acquises au cours de ses études odontologiques. Les organisateurs du stage à l'Unité de formation et de recherche (UFR) de ClermontFerrand ont choisi d'immerger les étudiants pendant deux mois à temps complet au sein d'un cabinet libéral. Un stage pilote avec sept étudiants et donc sept maitres de stage volontaires, avait été mis en place dès l'année universitaire 2006-2007 au sein de l'UFR.

L'objectif principal de ce travail était de mettre en évidence l'influence qu'a pu avoir ce stage sur le sentiment de compétence ou d'efficacité perçu par les étudiants. Nous comprenons cette forme autoévaluation au sens précisé par Dory, qui fait référence «au jugement porté sur ses propres compétences [ou sentiment d'efficacité personnelle (self efficacy belief)] et qui peut avoir lieu a posteriori, après l'exécution d'une action spécifique mais qui peut également prendre la forme d'une évaluation a priori de ses capacités ou de son potentiel à agir ${ }^{[5]}$.

Nous avons demandé à une promotion d'étudiants d'auto-évaluer leur sentiment de performance concernant certaines capacités ou habiletés propres au chirurgien-dentiste et ceci à trois reprises au cours du cursus : en fin de cinquième année (troisième année du deuxième cycle des études odontologiques DCEO3) et deux fois au cours de la sixième année (première année du troisième cycle des études odontologiques - TCEO1), avant de partir en stage et au retour du stage. Un objectif secondaire de l'étude était de vérifier la concordance de l'auto-évaluation des étudiants par rapport à l'évaluation externe de leurs maîtres de stage respectifs.

\section{Matériel et méthodes}

\section{Populations de l'étude}

Cette étude a été menée auprès des étudiants inscrits en DCEO3 (D3, $5^{\mathrm{e}}$ année) à l'UFR d'odontologie de Clermont-Ferrand lors de l'année universitaire 2006-2007 $(n=46)$ puis en TCEO1 (T1, $6^{\mathrm{e}}$ année) en 2007-2008 ( $\left.n=42\right)$.

Les étudiants ont évalué leur sentiment de compétence à trois reprises à l'aide d'un questionnaire, administré en fin d'année de D3 (juin 2007, questionnaire D3), avant le départ en stage actif en T1 (janvier 2008, questionnaire T1a) et au retour du stage actif (avril 2008 à juin 2008, questionnaire T1b).

Les praticiens ayant reçu des étudiants en stage actif $(n=42)$ ont également évalué le niveau d'efficacité qu'ils attribuaient à ces derniers à l'aide du même questionnaire.

\section{Élaboration du questionnaire}

Le questionnaire consiste en une auto-évaluation du sentiment de compétence par les étudiants ou en une évaluation de l'efficacité de l'étudiant par son maître de stage. Le questionnaire utilisé est celui déjà utilisé dans une étude précédente ${ }^{[7]}$. Le choix des capacités ou habiletés professionnelles explorées par le questionnaire a été fait parmi celles que doit posséder le futur odontologiste européen, telles qu'elles ont été élaborées à partir d'un consensus approuvé par l'ensemble des facultés de chirurgie dentaire membres de l'ADEE (Association for Dental Education in Europe $)^{[1]}$. Les capacités choisies relèvent des domaines suivants : professionnalisme, communication et capacités relationnelles, recueil des informations cliniques, restauration et entretien de la santé buccale, encouragement à la politique de santé publique, autres compétences (urgences dentaires et médicales, prescriptions, patients à besoins spécifiques). Au total 52 capacités ou habiletés professionnelles ont été retenues. 
Le questionnaire est composé de deux parties :

- Partie A (informations générales). Elle comprend quatre questions pour le questionnaire étudiant et trois pour celui du praticien. Pour le questionnaire «étudiant», les quatre questions concernaient son année de naissance, son sexe, un éventuel redoublement de D3 et l'existence de remplacement en fin de D3 et au cours de T1. En effet, dès lors qu'ils ont validé leur cinquième année, les étudiants sont autorisés à effectuer des remplacements au cours desquels ils exercent, contrairement au stage actif, sans la supervision d'un praticien. Le nombre d'équivalent-semaine (1 équivalent-semaine $=5$ jours) de remplacement devait être précisé en cas de réponse positive à cette dernière question. Pour les praticiens, les trois questions concernaient leur année de naissance, leur sexe, leur type d'exercice (omnipratique avec ou sans exercice prédominant).

- Partie B (capacités professionnelles). Elle permet l'évaluation (auto-évaluation de la part des étudiants et évaluation externe de la part des maîtres de stage) de 52 capacités ou habiletés professionnelles. Cette évaluation s'est faite au moyen d'une échelle visuelle analogique (EVA) non graduée allant de 0 (pas du tout compétent) à 10 (très compétent).

La mesure de la distance du trait vertical porté par le répondant sur l'échelle par rapport au point 0 donne un score compris entre 0 et 10 . Il est également possible de cocher une case «ne se prononce pas » pour chaque compétence. Le libellé des capacités ou habiletés est exactement le même dans les trois questionnaires distribués aux étudiants ainsi que dans celui adressé aux maîtres de stage. Un paragraphe expliquant comment utiliser l'EVA, accompagné d'un exemple, était placé en début de questionnaire.

- Une question ouverte permettant au répondant d'apporter des commentaires, terminait le questionnaire. Celui-ci était anonyme et l'étude a été menée sur la base du volontariat.

\section{Recueil et traitement des données}

Le questionnaire a été distribué aux étudiants lors d'un cours ou d'un séminaire. Ils pouvaient le remettre anonymement dans une boîte aux lettres prévue à cet effet. Les maîtres de stage ont reçu le questionnaire par la poste. Une lettre expliquant les objectifs de l'étude ainsi qu'une enveloppe prétimbrée pour le retour l'accompagnaient.

Les résultats ont été analysés de façon descriptive à l'aide du logiciel Excel 2007 et les analyses statistiques ont été réalisées au moyen du logiciel Statistical Package for Social Sciences (SPSS) ${ }^{\circledR 11.5}$. D'éventuelles différences entre les résultats des questionnaires D3, T1a et T1b ont été mises en évidence au moyen du test de Student Newman Keuls post Anova $(\alpha=0,05)$. La concordance des autoévaluations et des évaluations externes, respectivement effectuées en fin de stage actif par les étudiants et les maîtres de stage a été examinée à l'aide d'un test bilatéral de Dunnett, réalisé pour chaque capacité professionnelle. Nous avons comparé les scores des capacités professionnelles pour lesquelles la moitié au moins des maîtres de stage qui avaient répondu au questionnaire s'étaient prononcés. Ainsi, seuls les scores étudiants-maîtres de stage de 32 capacités sur les 52 présentes dans le questionnaire ont été comparés. Les 20 capacités ou habiletés qui ont été peu évaluées par les maîtres de stage, sont celles qui, en raison de leur rareté ou de leur complexité, sont peu sollicitées par les étudiants au cours de leur stage de deux mois.

\section{Résultats}

\section{Pourcentage de réponse et profil des répondants}

En ce qui concerne les étudiants, les taux de réponse étaient les suivants : 60,9\% pour le questionnaire D3 et, respectivement, 95,2 et $100 \%$ pour les questionnaires T1a et T1b (test du $\chi^{2}$ non significatif pour le critère sexuel). Soixante-neuf $\mathrm{p}$. cent des praticiens maîtres de stage ont renvoyé le questionnaire rempli. L'âge moyen de la promotion étudiée est, fin 2008 , de $25,5 \pm 1,1$ ans ; celui des maîtres de stage est de 48,6 \pm 9,7 ans ; 42,5\% des étudiants avaient déjà effectué des remplacements avant de partir en stage actif. La durée moyenne de ces remplacements est de 1,7 équivalent-semaine; 


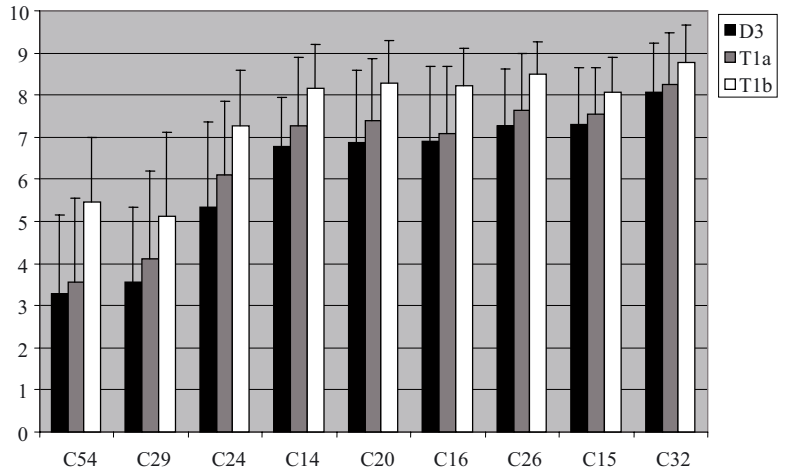

C54 : Diagnostiquer et prendre en charge les troubles de l'occlusion, les pathologies des ATM C29 : Prendre en charge les pathologies de la muqueuse buccale aigües et chroniques (traiter et adresser)

C24 : Elaborer un plan de traitement multidisciplinaire

C14 : Maintenir un environnement de travail sans danger pour les patients, l'équipe soignante et soi-même

$\mathrm{C} 20$ : Tenir des dossiers patients à jour (radios compte-rendus,...)

C16 : Savoir communiquer avec l'équipe soignante

C26 : Enseigner au patient les principes de la santé bucco-dentaire, d'hygiène, d'alimentation, évaluer le risque carieux

C15 : Savoir communiquer avec ses patients (évaluer leurs attentes et leur motivation, obtenir un consentement éclairé, gérer leurs appréhensions et leur stress...)

C32 : Pratiquer le détartrage, surfaçage.

Fig. 1. Capacités professionnelles pour lesquelles le sentiment de compétence des étudiants est améliorée à l'issue du stage actif chez le praticien. D3 : troisième année du deuxième cycle des études odontologiques. T1a : première année du troisième cycle des études odontologiques, avant le départ en stage actif. T1b : première année du troisième cycle des études odontologiques, au retour du stage actif.

$54,8 \%$ des étudiants avaient effectué des remplacements lorsqu'ils ont rempli le questionnaire après le stage actif. La durée moyenne des remplacements était alors de 2,9 équivalent-semaine.

\section{Impact du stage actif sur le sentiment}

\section{de compétence}

Nous avons pu déterminer cinq profils de capacités professionnelles et, tout d'abord, celles pour lesquelles les étudiants ont le sentiment d'avoir amélioré leur compétence grâce au stage actif (figure 1). Ce sont les capacités ou habiletés suivantes : «Maintenir un environnement de travail sans danger pour les patients, l'équipe soignante et soi-même » (C14), «Savoir communiquer avec ses patients » (C15), « « Savoir communiquer avec l'équipe soignante» (C16), « Tenir des dossiers patients à jour » (C20),

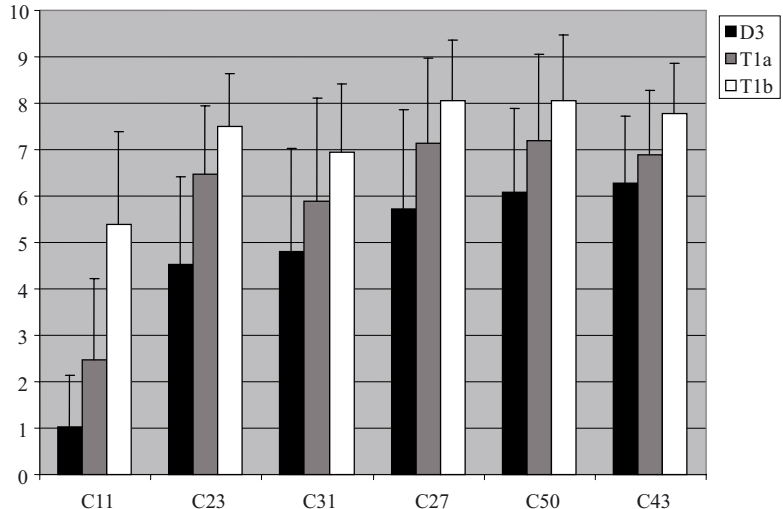

C11 : Gérer la structure du cabinet et en particulier tenir une comptabilité

C23 : Connaître et savoir prescrire des examens complémentaires

C31 : Évaluer l'état parodontal initial, faire un diagnostic et établir un plan de traitement global

C27 : Pratiquer les actes courants de prévention (fluoration, sealants)

C50 : Connaître les indications et les contre-indications d'un traitement implantaire

C43 : Prendre en charge la douleur des patients, maitriser les indications et les techniques d'anesthésie et d'analgésie.

Fig. 2. Capacités professionnelles pour lesquelles le sentiment de compétence des étudiants s'améliore avec le temps et/ou à l'issue du stage actif. D3 : troisième année du deuxième cycle des études odontologiques. T1a : première année du troisième cycle des études odontologiques, avant le départ en stage actif. $\mathrm{T} 1 \mathrm{~b}$ : première année du troisième cycle des études odontologiques, au retour du stage actif.

«Élaborer un plan de traitement multidisciplinaire » (C24), «Enseigner au patient les principes de la santé bucco-dentaire, d'hygiène, d'alimentation, évaluer le risque carieux » (C26), « Prendre en charge les pathologies de la muqueuse buccale aiguës et chroniques » (C29), «Pratiquer le détartrage, surfaçage » $(\mathrm{C} 32)$ et «Diagnostiquer et prendre en charge les troubles de l'occlusion, les pathologies des ATM» (C54). Il n'existe pas de différence significative entre la moyenne des scores des questionnaires D3 et T1a pour ces capacités ou habiletés; par contre on note une différence significative entre le score $\mathrm{T} 1 \mathrm{~b}$ et les deux autres scores.

D'autres capacités professionnelles ont peutêtre également vu leur sentiment d'acquisition amélioré par le stage actif puisque l'on note une différence significative entre le score $\mathrm{T} 1 \mathrm{a}$ et $\mathrm{T} 1 \mathrm{~b}$ mais il existe aussi un effet du temps sur leur acquisition puisque les scores D3 et T1a sont également significativement différents (figure 2). Ce sont les 
Tableau I. Capacités professionnelles dont les scores moyens en troisième année du deuxième cycle des études odontologiques (D3), en première année du troisième cycle des études odontologiques, avant le départ en stage actif (T1a) et en première année du troisième cycle des études odontologiques, au retour du stage actif ( $\mathrm{T} 1 \mathrm{~b})$ ne présentent pas de différence significative.

\begin{tabular}{|c|c|c|c|}
\hline Capacités professionnelles & D3* & T1a* & T1b* \\
\hline Effectuer une biopsie, une exérèse $\left(\mathrm{C} 30^{* *}\right)$ & $1,24 \pm 1,51$ & $1,56 \pm 1,89$ & $2,21 \pm 2,13$ \\
\hline Effectuer la mise en place chirurgicale d'implants (C51) & $1,66 \pm 1,65$ & $2,30 \pm 2,11$ & $2,83 \pm 2,13$ \\
\hline $\begin{array}{l}\text { Effectuer les chirurgies parodontales (lambeau d'assainissement...) } \\
\text { (C33) }\end{array}$ & $3,81 \pm 1,73$ & $4,39 \pm 2,89$ & $5,15 \pm 2,38$ \\
\hline Réaliser une prothèse fixée plurale (C46) & $6,76 \pm 1,57$ & $7,01 \pm 1,94$ & $7,64 \pm 1,24$ \\
\hline Réaliser une prothèse provisoire $(\mathrm{C} 49)$ & $7,09 \pm 2,38$ & $7,46 \pm 1,75$ & $8,00 \pm 1,39$ \\
\hline $\begin{array}{l}\text { Proposer au patient les différentes thérapeutiques prothétiques adaptées à } \\
\text { son cas (C44) }\end{array}$ & $7,19 \pm 1,71$ & $7,45 \pm 1,39$ & $7,91 \pm 1,16$ \\
\hline Réaliser une prothèse totale amovible $(\mathrm{C} 48)$ & $7,31 \pm 1,86$ & $7,87 \pm 1,63$ & $7,80 \pm 1,51$ \\
\hline Réaliser un retraitement endodontique (C37) & $7,52 \pm 1,42$ & $7,72 \pm 1,28$ & $7,96 \pm 1,03$ \\
\hline $\begin{array}{l}\text { Réaliser le traitement endodontique quelle que soit la dent concernée (in- } \\
\text { cisive, molaire...) (C36) }\end{array}$ & $7,52 \pm 1,47$ & $8,10 \pm 0,96$ & $7,97 \pm 1,22$ \\
\hline Réaliser une prothèse partielle amovible (C47) & $7,66 \pm 1,47$ & $8,16 \pm 1,08$ & $8,20 \pm 0,99$ \\
\hline Réaliser une prothèse fixée unitaire (C45) & $7,88 \pm 1,41$ & $8,29 \pm 0,83$ & $8,43 \pm 0,75$ \\
\hline $\begin{array}{l}\text { Traiter la maladie carieuse, restaurer les dents et la denture en terme de } \\
\text { fonction mais aussi d'esthétique (C } 35)\end{array}$ & $8,10 \pm 1,33$ & $8,28 \pm 0,99$ & $8,59 \pm 0,74$ \\
\hline
\end{tabular}

D3 : troisième année du deuxième cycle des études odontologiques

T1a : première année du troisième cycle des études odontologiques, avant le départ en stage actif

$\mathrm{T} 1 \mathrm{~b}$ : première année du troisième cycle des études odontologiques, au retour du stage actif

* D3 : moyenne ( \pm écart-type) des scores obtenus en fin de cinquième année (D3); T1a : moyenne ( \pm écart-type) des scores obtenus en sixième année (T1) avant de partir en stage actif; T1b : moyenne ( \pm écart-type) des scores obtenus en sixième année après le stage actif

** Cx : Capacité $n^{\circ} \mathrm{x}$.

capacités ou habiletés : «Gérer la structure du cabinet et en particulier tenir une comptabilité » (C11), «Connaître et savoir prescrire des examens complémentaires » (C23), «Évaluer l'état parodontal initial, faire un diagnostic et établir un plan de traitement global » $(\mathrm{C} 31)$, « Pratiquer les actes courants de prévention (fluoration, sealants)» (C27), «Connaître les indications et les contre-indications d'un traitement implantaire » (C50) et « Prendre en charge la douleur des patients, maîtriser les indications et les techniques d'anesthésie et d'analgésie » (C43).

On ne note pas d'effet du stage actif pour les autres capacités professionnelles. Le tableau I montre les scores de celles dont le sentiment d'acquisition s'améliore très peu avec le temps. Il n'existe pas de différence significative entre les trois scores D3, T1a et T1b. Le tableau II montre les capacités professionnelles pour lesquelles il existe une différence significative entre le score de D3 et les deux scores de T1 mais pas entre les scores T1a et T1b. Enfin, le dernier profil de capacités professionnelles est représenté par celles pour lesquelles on ne note pas de différence significative entre les scores D3-T1a et T1a-T1b. Par contre, une différence significative existe entre les scores D3 et T1b (tableau III).

\section{Concordance d'appréciation du sentiment de compétence entre stagiaires et maîtres de stage}

Il n'existe pas de différence significative d'appréciation entre stagiaires et maîtres de stage pour 
Tableau II. Capacités professionnelles pour lesquelles il existe une différence significative entre le score de troisième année du deuxième cycle des études odontologiques (D3) et ceux de première année du troisième cycle des études odontologiques avant le départ en stage actif ( $\mathrm{T} 1 \mathrm{a})$ et de première année du troisième cycle des études odontologiques au retour du stage actif (T1b).

\begin{tabular}{|c|c|c|c|}
\hline Capacités professionnelles & D3 & T1a & $\mathrm{T} 1 \mathrm{~b}$ \\
\hline $\begin{array}{l}\text { Reconnaître les signes d'abus, maltraitance et abandon } \\
\text { envers les patients et savoir en informer les instances } \\
\text { légales compétentes (C25) }\end{array}$ & $3,28 \pm 2,03$ & $4,43 \pm 2,08$ & $5,13 \pm 1,94$ \\
\hline $\begin{array}{l}\text { Diagnostiquer les problèmes maxillo-faciaux notamment } \\
\text { les douleurs chroniques oro-faciales et savoir au besoin } \\
\text { adresser à un confrère (C56) }\end{array}$ & $3,43 \pm 1,62$ & $4,45 \pm 1,96$ & $5,22 \pm 1,83$ \\
\hline Prendre en charge les traumatismes alvéolo-dentaires (C41) & $3,44 \pm 2,18$ & $5,17 \pm 2,59$ & $5,96 \pm 2,20$ \\
\hline $\begin{array}{l}\text { Diagnostiquer les pathologies de la muqueuse } \\
\text { buccale aigües et chroniques (C28) }\end{array}$ & $3,81 \pm 1,85$ & $4,71 \pm 1,84$ & $5,33 \pm 1,69$ \\
\hline $\begin{array}{l}\text { Réaliser une avulsion complexe (dent incluse, } \\
\text { alvéolectomie...) (C40) }\end{array}$ & $4,05 \pm 2,29$ & $5,35 \pm 2,79$ & $6,27 \pm 2,51$ \\
\hline $\begin{array}{l}\text { Prendre en charge les complications per et post opératoires } \\
\text { de la chirurgie (C42) }\end{array}$ & $4,11 \pm 2,31$ & $5,98 \pm 2,16$ & $6,83 \pm 1,89$ \\
\hline $\begin{array}{l}\text { Connaître les dangers des radiations ionisantes } \\
\text { et appliquer les règles de protection }(\mathrm{C} 21)\end{array}$ & $4,97 \pm 2,36$ & $7,19 \pm 1,85$ & $7,70 \pm 1,46$ \\
\hline $\begin{array}{l}\text { Diagnostiquer les troubles orthodontiques et savoir } \\
\text { adresser à un spécialiste (C55) }\end{array}$ & $5,19 \pm 1,72$ & $6,22 \pm 1,72$ & $6,76 \pm 1,69$ \\
\hline $\begin{array}{l}\text { Prescrire les médications courantes en odontologie } \\
\text { en fonction des pathologies présentes et des traitements } \\
\text { en cours des patients, y compris chez l'enfant (C62) }\end{array}$ & $5,96 \pm 2,11$ & $7,61 \pm 0,99$ & $7,73 \pm 1,13$ \\
\hline Gérer l'urgence dentaire (C60) & $6,19 \pm 1,63$ & $7,45 \pm 1,13$ & $8,00 \pm 0,94$ \\
\hline Réaliser un examen clinique extra et intra buccal complet (C19) & $6,39 \pm 1,83$ & $7,55 \pm 1,03$ & $7,99 \pm 0,99$ \\
\hline Prendre et interpréter des clichés radiologiques (C22) & $6,79 \pm 1,38$ & $7,62 \pm 1,08$ & $8,03 \pm 0,94$ \\
\hline $\begin{array}{l}\text { Appliquer les principes de déontologie vis-à-vis du patient } \\
\text { (éthique, intégrité, honnêteté...) (C12) }\end{array}$ & $7,14 \pm 1,89$ & $8,13 \pm 0,97$ & $8,21 \pm 1,14$ \\
\hline $\begin{array}{l}\text { Savoir reconnaître ses limites (demander conseil } \\
\text { ou adresser un patient à un confrère) }(\mathrm{C} 13)\end{array}$ & $7,18 \pm 1,49$ & $8,10 \pm 1,18$ & $8,28 \pm 0,93$ \\
\hline $\begin{array}{l}\text { Conduire un entretien médical complet, recueillir l'histoire } \\
\text { dentaire et médicale du patient (C18) }\end{array}$ & $7,24 \pm 1,53$ & $8,05 \pm 0,92$ & $8,38 \pm 0,75$ \\
\hline Réaliser l'avulsion simple d'une racine ou d'une dent sur arcade (C39) & $7,88 \pm 1,38$ & $8,67 \pm 0,74$ & $8,78 \pm 0,97$ \\
\hline Diagnostiquer la maladie carieuse et adapter sa prise en charge (C34) & $8,00 \pm 1,38$ & $8,48 \pm 0,86$ & $8,68 \pm 0,78$ \\
\hline
\end{tabular}

D3 : troisième année du deuxième cycle des études odontologiques

(T1a) : première année du troisième cycle des études odontologiques avant le départ en stage actif

(T1b) : première année du troisième cycle des études odontologiques au retour du stage actif

* D3 : moyenne ( \pm écart-type) des scores obtenus en fin de cinquième année (D3); T1a : moyenne ( \pm écart-type) des scores obtenus en sixième année (T1) avant de partir en stage actif; T1b : moyenne ( \pm écart-type) des scores obtenus en sixième année après le stage actif

** Cx : Capacité $\mathrm{n}^{\circ} \mathrm{x}$.

27 des 32 capacités professionnelles étudiées. Par contre, cinq ont été sur-évaluées par les stagiaires par rapport aux maîtres de stage (figure 3). Ce sont les capacités ou habiletés suivantes : gérer la structure du cabinet et en particulier tenir une comptabilité (C11), savoir communiquer avec ses patients (évaluer leurs attentes et leur motivation, obtenir un consentement éclairé, gérer leurs appréhensions et leur stress...) (C15), conduire un entretien médical complet, recueillir l'histoire dentaire et 
Tableau III. Capacités professionnelles pour lesquelles il existe une différence significative entre les scores D3 et T1 après stage actif mais pas entre D3-T1a et T1a-T1b.

\begin{tabular}{|c|c|c|c|}
\hline Capacités professionnelles & D3 & T1a & T1b \\
\hline Réaliser une prothèse supra-implantaire (C52) & $2,35 \pm 2,55$ & $3,00 \pm 2,96$ & $4,30 \pm 2,68$ \\
\hline $\begin{array}{l}\text { Reconnaître les manifestations orales des principales pathologies géné- } \\
\text { rales (C57) }\end{array}$ & $3,39 \pm 1,76$ & $3,97 \pm 1,92$ & $4,67 \pm 1,88$ \\
\hline Gérer l'urgence médicale (C61) & $3,78 \pm 1,57$ & $4,62 \pm 1,67$ & $5,18 \pm 2,06$ \\
\hline $\begin{array}{l}\text { Prendre en charge les patients à besoins spécifiques (personnes dépen- } \\
\text { dantes, âgées...) (C63) }\end{array}$ & $4,55 \pm 2,16$ & $5,53 \pm 2,05$ & $5,87 \pm 1,96$ \\
\hline $\begin{array}{l}\text { Poser les indications de chirurgie endodontique et savoir adresser si be- } \\
\text { soin (C38) }\end{array}$ & $4,68 \pm 2,23$ & $5,69 \pm 2,57$ & 2,17 \\
\hline $\begin{array}{l}\text { Faire la promotion de la santé et la prévention des maladies générales } \\
\text { (C59) }\end{array}$ & $5,50 \pm 2,42$ & $5,88 \pm 2,13$ & $6,91 \pm 1,75$ \\
\hline $\begin{array}{l}\text { Maîtriser les technologies modernes d'information pour la recherche, la } \\
\text { documentation, la formation continue ou la communication (C17) }\end{array}$ & $5,85 \pm 2,85$ & $6,57 \pm 1,93$ & $7,50 \pm 1,58$ \\
\hline $\begin{array}{l}\text { Savoir prendre en charge le traitement d'un enfant, quel que soit son âge, } \\
\text { en termes de communication, relation, apprentissage et acceptation des } \\
\text { soins (C53) }\end{array}$ & $6,47 \pm 1,65$ & $6,97 \pm 1,49$ & $7,42 \pm 1,18$ \\
\hline
\end{tabular}

D3 : troisième année du deuxième cycle des études odontologiques

(T1a) : première année du troisième cycle des études odontologiques avant le départ en stage actif

(T1b) : première année du troisième cycle des études odontologiques au retour du stage actif

* D3 : moyenne ( \pm écart-type) des scores obtenus en fin de cinquième année (D3); T1a : moyenne ( \pm écart-type) des scores obtenus en sixième année (T1) avant de partir en stage actif; T1b : moyenne ( \pm écart-type) des scores obtenus en sixième année après le stage actif

** Cx : Capacité $n^{\circ} \mathrm{x}$.

médicale du patient (C18), réaliser l'avulsion simple d'une racine ou d'une dent sur arcade (C39) et proposer au patient les différentes thérapeutiques prothétiques adaptées à son cas (C44).

\section{Question ouverte}

Les taux de réponse à la question ouverte de fin de questionnaire sont respectivement de 21,4; 32,5; 19 et $27,6 \%$ pour les questionnaires D3, T1a, T1b et maîtres de stage. Les remarques exprimées par les répondants ont permis d'étayer la discussion de cet article.

\section{Discussion}

L'objectif de cette étude était de documenter l'apport du stage actif chez le praticien sur le sentiment de compétence professionnelle perçu par l'étudiant en chirurgie dentaire. Au cours de ses études, ce dernier acquiert ses futures compétences, notamment en pratiquant des actes courants sur des patients au sein d'un service hospitalier d'odontologie, modèle de travail qui est différent d'un cabinet libéral, futur lieu d'exercice professionnel de la très grande majorité des étudiants. Une immersion au sein d'un cabinet dentaire au sein duquel l'étudiant exerce son art en autonomie contrôlée avec le soutien d'un maître de stage peut donc permettre une transition douce entre ces deux types de structures. De plus, le stage au sein d'un cabinet libéral peut permettre à l'étudiant d'acquérir certaines capacités ou habiletés qu'il est difficile de maîtriser dans une structure hospitalière. Évaluer l'apport de ce stage rentre donc dans une démarche qualité visant à améliorer la formation donnée au sein de notre UFR.

Le pourcentage de réponses aux questionnaires est relativement important puisqu'il se situe entre 61 et $100 \%$ selon les différentes périodes pour la promotion étudiée. Il atteint $69 \%$ pour les maîtres 


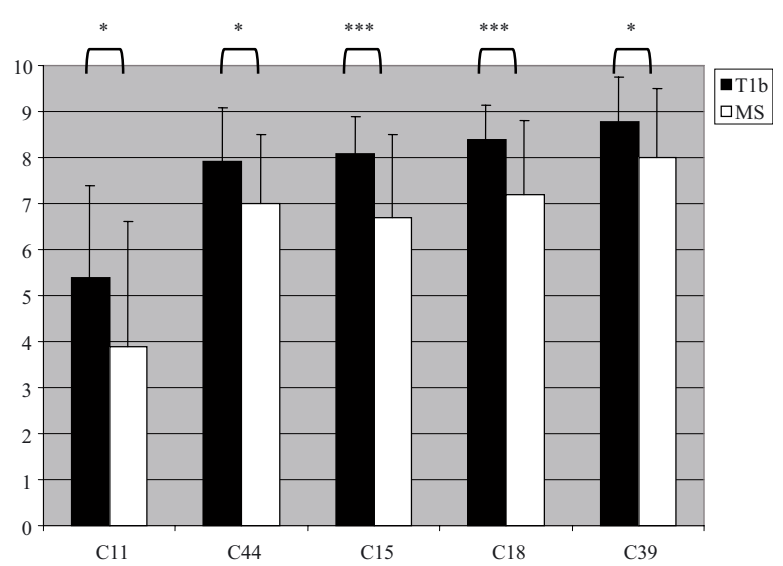

C11 : Gérer la structure du cabinet et en particulier tenir une comptabilité C44 : Proposer au patient les différentes thérapeutiques prothétiques adaptées à son cas C15 : Savoir communiquer avec ses patients (évaluer leurs attentes et leur motivation, obtenir un consentement éclairé, gérer leurs appréhensions et leur stress...)

C18 : Conduire une entretien médical complet, recueillir l'histoire dentaire et médicale du patient

C39 : Réaliser l'avulsion simple d'une racine ou d'une dent sur arcade

* La différence est significative : $p<0,05$; *** La différence est significative : $p<0,001$

Fig. 3. Capacités professionnelles dont les scores d'évaluation diffèrent significativement entre les étudiants de première année du troisième cycle au retour du stage actif ( $\mathrm{T} 1 \mathrm{~b})$ et les maîtres de stages (MS).

de stage. Ces résultats sont supérieurs à ceux obtenus lors d'autres études ${ }^{[8,9]}$. Nos résultats sont représentatifs des différentes populations considérées puisque le test du $\chi^{2}$ s'est révélé non significatif en ce qui concerne le critère sexuel.

Bien que nous n'ayons pas démontré les qualités psychométriques de validité et de fidélité de notre questionnaire par rapport au référentiel de compétences à évaluer, nous postulons que le mode de construction de ce questionnaire, à partir d'un répertoire de compétences établi à l'issue d'une recherche méthodique de consensus, lui confère une certaine validité de construit. Cette limite étant admise, l'évaluation du sentiment de compétence au moyen d'une EVA permet d'attribuer une valeur numérique au sentiment de compétence perçu par rapport à chaque capacité ou habileté et peut permettre une analyse plus fine des résultats, ce qui n'est pas toujours possible avec d'autres types d'échelles comme celles de type Likert à plusieurs niveaux, souvent utilisées lors d'études comparables ${ }^{[6,10,11]}$. Bien que certaines études dans le domaine de l'odontologie tendent à affirmer que le processus d'auto-évaluation pourrait offrir un certain intérêt dans les mécanismes d'apprentissage de l'étudiant ${ }^{[12,13]}$, des revues de synthèse récentes montrent que la capacité d'autoévaluation des étudiants et des professionnels de santé est médiocre ${ }^{[5,14]}$. Il est aujourd'hui recommandé de combiner les résultats de l'auto-évaluation avec ceux d'une rétroaction externe afin de mieux orienter les apprentissages et d'optimiser les pratiques $^{[5]}$. Lors de notre étude, nous avons tenté d'évaluer la capacité des étudiants à s'auto-évaluer en comparant les scores qu'ils s'attribuaient en fin de stage par rapport à ceux que leur attribuaient leurs maîtres de stage. Pour 27 des capacités ou habiletés sur les 32 comparées, il n'existait pas de différence significative entre les deux scores; pour autant, nous ne pouvons porter aucune conclusion sur l'aptitude de nos étudiants à s'évaluer à leur juste valeur puisque notre étude mesure la capacité d'auto-évaluation au niveau du groupe et non de l'individu ${ }^{[15]}$. De même, la validité et la fidélité de l'évaluation externe faite par les maîtres de stage n'ont pas été étudiées au cours de notre étude. Une étude de suivi, étudiant par étudiant, pourrait permettre d'évaluer individuellement les processus d'auto-évaluation et d'évaluation externe et de mettre plus précisément en évidence la capacité de nos étudiants à s'auto-évaluer. Notre étude ne permet donc pas de mettre en évidence un impact du stage actif sur l'acquisition de capacités ou d'habiletés professionnelles mais seulement une influence positive de ce stage sur le sentiment de compétence que perçoivent les étudiants vis-à-vis de ces capacités ou habiletés.

Une fois qu'ils ont validé le CSCT (certificat de synthèse clinique et thérapeutique) en fin de cinquième année (D3), les étudiants sont autorisés à effectuer des remplacements. L'amélioration éventuelle du sentiment de compétence au cours de ces périodes d'exercice en cabinet libéral pourrait entrer en ligne de compte lors de notre processus d'autoévaluation. C'est pourquoi, nous avons demandé aux 
étudiants, grâce aux différents questionnaires, s'ils avaient effectué des remplacements et quelle avait été leur durée en équivalent-semaine. On note que plus de $42 \%$ ont déjà remplacé avant de partir en stage actif et ce pour une durée moyenne de 1,7 semaines, soit environ 8 jours. Après le stage actif, près de $55 \%$ des étudiants en ont effectué, avec une durée moyenne de 2,9 équivalents-semaine, soit 14 jours. Ces différentes périodes de remplacement participent donc peu à l'amélioration du sentiment de compétence au cours des deux dernières années d'études. De plus, si on considère que le stage actif dure neuf semaines et qu'en moyenne les étudiants ont effectué six jours de remplacement entre les questionnaires T1a et T1b, on peut donc juger comme accessoire l'impact qu'auraient pu avoir ces jours de remplacement par rapport à celui du stage actif dans le processus d'auto-évaluation.

Notre étude a permis de mettre en évidence une amélioration, après le stage actif, du sentiment de compétence concernant neuf capacités professionnelles (C14, C15, C16, C20, C24, C26, C29, C32 et C54). Cette amélioration s'explique très probablement, pour certaines d'entre elles, par la différence de fonctionnement entre la structure hospitalière, au sien de laquelle l'étudiant reçoit sa formation clinique, et le cabinet libéral. À l'hôpital, une partie des tâches est effectuée par un personnel qualifié et les étudiants y sont très peu acteurs. C'est l'exemple de la gestion administrative des patients, de la stérilisation, du tri et de l'élimination des déchets, de la tenue du dossier patient, de la gestion des différents personnels et des relations que l'on entretient avec. Ceci peut donc expliquer l'effet bénéfique du stage actif sur des capacités telles que «Maintenir un environnement de travail sans danger pour les patients, l'équipe soignante et soi-même » (C14), « Savoir communiquer avec l'équipe soignante et avec ses patients » $(\mathrm{C} 15$ et $\mathrm{C} 16)$ ou « Tenir des dossiers patients à jour » (C20).

Au cours du stage en service hospitalier d'odontologie, une partie des patients, dont les problèmes dentaires relèvent d'un certain degré de «spécialisation », sont vus par des praticiens en consultation (appelée « soins directs »), à laquelle les étudiants ont peu accès. Ainsi les capacités ou habiletés «Prendre en charge les pathologies de la muqueuse buccale aiguës et chroniques » (C29) et « Diagnostiquer et prendre en charge les troubles de l'occlusion, les pathologies des articulations temporomandibulaires (ATM)» (C54) ont vu leur score fortement augmenter après le stage actif puisque les étudiants ont pu être confrontés à ce profil de patient au cours de leur stage. En ce qui concerne la dernière capacité (C54), il semblerait que la progression de son score ne soit pas seulement due au stage actif lui-même mais aussi aux cours théoriques d'occlusodontologie, placés en T1, et dont l'évaluation se situait en fin d'année. Les révisions préparant les étudiants à l'épreuve finale écrite dans cette matière se déroulaient en même temps que le stage actif. De plus, cette capacité a également été reconnue comme difficile à acquérir dans d'autres études ${ }^{[16]}$ et requiert un minimum d'expérience pour être parfaitement acquise.

«Élaborer un plan de traitement multidisciplinaire » (C24) est une des capacités dont le sentiment de maîtrise a également été fortement amélioré après le stage actif. Ceci peut encore s'expliquer par la différence d'organisation de travail entre le service hospitalier et un cabinet dentaire. En effet, dans la première structure, le plan de traitement est élaboré par l'étudiant avec l'aide d'un enseignant ou d'un groupe d'enseignants, lors de la première visite de chaque patient, puis la mise en œuvre de la thérapeutique est organisée en disciplines. Ceci fait que l'étudiant a du mal à faire le lien entre les différentes phases du traitement et à anticiper ses étapes. De plus, le plan de traitement est susceptible d'évoluer au cours des séances du fait de l'intervention d'enseignants différents et il peut arriver que plusieurs étudiants participent aux soins d'un même patient. Ceci devrait donc sensibiliser le corps enseignant à modifier les techniques d'apprentissage pratiquées au sein du service afin de rendre beaucoup plus évidente pour l'étudiant la vision globale du patient. En 2006, Patel ${ }^{[10]}$ avait déjà remarqué, dans une étude similaire à la nôtre, que $56 \%$ des maîtres 
de stage avaient estimé que cette capacité était insuffisamment enseignée au cours des années d'études.

Le stage chez le praticien a également pu augmenter le sentiment de compétence des étudiants vis-à-vis des capacités ou habiletés professionnelles C11, C23, C27, C31, C43 et C50 mais ceci est plus difficile à déterminer puisque le score obtenu pour ces capacités professionnelles a également progressé entre D3 et T1. C'est le cas par exemple de la capacité «Gérer la structure d'un cabinet et en particulier tenir une comptabilité » (C11). Les étudiants suivent l'enseignement de vie professionnelle lors du premier semestre de l'année de T1. Il est donc logique de voir la note de cette capacité professionnelle nettement s'améliorer entre la fin de D3 et le premier questionnaire de T1. Son score progresse également après le stage actif. Les étudiants sont généralement peu confrontés aux problèmes de gestion et de comptabilité au centre de soins. Il est donc normal qu'après le stage actif, au cours duquel l'étudiant a été sensibilisé à cet aspect de la profession, le sentiment de compétence vis-à-vis de cette capacité se soit amélioré. Cependant, le score obtenu reste faible, même après le stage, et c'est une capacité qui est toujours identifiée, dans des études semblables à la nôtre, comme difficilement acquise en fin de formation initiale et même après quelques années d'exercice $^{[7-9,16]}$.

Le sentiment de compétence vis-à-vis des autres capacités ou habiletés professionnelles ne semble donc pas avoir été amélioré par le stage d'immersion en milieu professionnel. Cependant, des profils différents émergent selon les scores obtenus. Pour certaines capacités ou habiletés, le sentiment de compétence augmente logiquement au fur et à mesure que les étudiants acquièrent de l'expérience. Pour d'autres, le sentiment d'efficacité personnelle est élevé dès la fin de D3 et, logiquement, s'améliore très peu pendant la dernière année d'études. Ceci tendrait donc à démontrer que certaines capacités ou habiletés pourraient s'acquérir beaucoup plus facilement que d'autres. C'est par exemple le cas du traitement de la maladie carieuse qui obtient également un très bon score dans des études similaires ${ }^{[8,17-19]}$.
De plus certaines de ces capacités ou habiletés professionnelles sont abordées précocement au cours du cursus : l'étudiant acquiert les bases de sa gestuelle au cours des travaux pratiques (TP) dès la troisième année et ces notions sont ensuite mises en pratique lors de la clinique jusqu'en sixième année.

D'autres capacités ou habiletés obtiennent des scores très faibles qui ne s'améliorent pas ou très peu au cours du cursus. C'est le cas par exemple des capacités «Effectuer une biopsie, une exérèse » (C30) et «Diagnostiquer les pathologies de la muqueuse buccale aigües et chroniques »(C28). Le chirurgien-dentiste joue en effet un rôle important dans le dépistage de tumeurs de la cavité buccale. Ces capacités professionnelles semblent difficiles à acquérir mais de nombreuses autres études montrent qu'il devrait y avoir une meilleure formation dans la réalisation de ces actes ${ }^{[20]}$ et que l'aptitude à diagnostiquer des lésions précancéreuses n'est pas suffisante chez les étudiants en chirurgie dentaire ${ }^{[21,22]}$.

L'implantologie est une discipline relativement nouvelle dans le domaine de l'odontologie. Les capacités ou habiletés «Effectuer la pose chirurgicale d'implants » (C51) et «Réaliser une prothèse supra-implantaire » (C52) ont obtenu des scores peu élevés dans notre étude. Cependant, le sentiment de compétence des étudiants vis-à-vis de la capacité «Connaître les indications et contre-indications d'un traitement implantaire » est élevé, ce qui tend à montrer que l'enseignement théorique dispensé dans cette discipline est satisfaisant. L'enseignement de l'implantologie est désormais présent dans la majorité des facultés de chirurgie dentaire européennes mais les disparités en temps, moyens et méthodes d'apprentissage sont grandes ${ }^{[23,24]}$. Vu la place croissante de l'implantologie dans la pratique professionnelle depuis quelques années, il semble désormais nécessaire d'inclure dans le cursus une formation pratique à la prothèse supra-implantaire. Les compétences du futur chirurgien dentiste devant être élaborées en fonction des besoins en santé de la population, on peut également imaginer que la pose chirurgicale d'implants deviendra bientôt 
une capacité professionnelle à acquérir également au cours des années d'études.

\section{Conclusion}

Notre travail suggère de réels effets positifs du stage actif sur le sentiment de compétence ou d'efficacité personnelle de nos étudiants. Le service d'odontologie du CHRU de Clermont-Ferrand est un formidable outil d'enseignement et d'apprentissage mais son mode de fonctionnement ne correspond pas complètement à l'exercice futur de la majorité des étudiants. Le stage actif met en contact l'étudiant avec le milieu professionnel et lui permet de comprendre certaines notions telles que la gestion financière et administrative, la comptabilité, la stérilisation, l'élaboration de plans de traitement multidisciplinaires, qui jusqu'alors lui étaient presque inconnues. Il permet à l'étudiant d'augmenter son sentiment de compétence vis-à-vis de capacités ou d'habiletés difficilement acquises en milieu hospitalier et est donc indispensable à une formation initiale de qualité.

\section{Contributions}

Hélène Baudin et Valérie Roger-Leroi ont conçu le protocole de recherche, recueilli les résultats, interprété les données et rédigé le manuscrit. Emmanuel Nicolas a participé à la conception du protocole de recherche, a fait l'analyse statistique des données et a révisé le manuscrit. Bernard Chaumeil est responsable de l'organisation et du suivi du stage actif et a révisé le manuscrit.

\section{Remerciements}

Les auteurs tiennent à remercier chaleureusement les étudiants et les maîtres de stage qui ont accepté de participer à l'étude. Ils tiennent également à remercier le Doyen Thierry Orliaguet et l'ensemble des membres de l'UIPS de l'UFR d'odontologie de
Clermont-Ferrand, qui se sont tant battus pour que ce stage actif devienne réalité.

Ce travail a fait l'objet de la thèse pour le diplôme d'état de docteur en chirurgie dentaire d'Hélène Baudin.

\section{Références}

1. Plasschaert AJ, Holbrook WP, Delap E, Martinez C, Walmsley AD. Profile and competences for the European dentist. Eur J Dent Educ 2005;9:98-107.

2. Seth-Smith A. The role of vocational training in graduate education. Proceedings of the 31st annual meeting of ADEE. Eur J Dent Educ 2006;10:105.

3. Cabot LB, Patel HM. Aspects of the dental vocational training experience in the South East of England. $\mathrm{Br}$ Dent J 2007;202:278-9.

4. Clow R, Mehra S. Evaluation of vocational training of dentists in three different regions. Br Dent J 2006; 201:7748.

5. Dory V, de Foy T, Degryse J. L'auto-évaluation : postulat préalable, finalité de la mission éducative ou utopie pédagogique ? Clarifications conceptuelles et pistes pour une application en éducation médicale. Pédagogie Médicale 2009;10:41-53

6. Bartlett DW, Coward PY, Wilson R, Goodsman D, Darby J. Experiences and perceptions of vocational training reported by the 1999 cohort of vocational dental practitioners and their trainers in England and Wales. Br Dent J 2001;191:265-270.

7. Nicolas E, Baptiste M, Roger-Leroi V. Clermont-Ferrand dental school'curriculum: an appraisal by last-year students and graduates Eur J Dent Educ 2009;13:93-9.

8. Levy G, d'Ivernois JF, Brun D, Gagnayre R. A French dental school programme appraisal by alumni of 5-9 years standing. Eur J Dent Educ 1997;1:70-7.

9. Murray FJ, Blinkhorn AS, Bulman J. An assessment of the views held by recent graduates on their undergraduate course. Eur J Dent Educ 1999;3:3-9.

10. Patel J, Fox K, Grieveson B, Youngson CG. Undergraduate training as preparation for vocational training in England: a survey of vocational dental practitioners' and their trainers' views. Br Dent J 2006, suppl:9-15.

11. Sutton F, Ellituv ZN, Seed R. A survey of self-perceived educational needs of general dental practitioners in the Merseyside region. Prim Dent Care 2005;12:78-82.

12. Lindemann RA, Jedrychowski J. Self-assessed clinical competence: a comparison between students in an advanced dental education elective and in the general clinic. Eur J Dent Educ 2002;6:16-21. 
13. Zick A, Granieri M, Makoul G. First-year medical students' assessment of their own communication skills: a video-based, open-ended approach. Patient Educ Couns 2007;68:161-6.

14. Davis DA, Mazmanian PE, Fordis M, Van Harrison R, Thorpe KE, Perrier L. Accuracy of physician selfassessment compared with observed measures of competence: a systematic review. JAMA 2006;296:1094-102

15. Ward M, Gruppen L, Regehr G. Measuring selfassessment: current state of the art. Adv Health Sci Educ Theory Pract 2002;7:63-80

16. Greenwood LF, Lewis DW, Burgess RC. How competent do our graduates feel ? J Dent Educ 1998;62:304-13.

17. Raffek RN, Marchan SM, Naidu RS, Carotte PV. Perceived competency at graduation among dental alumni of the University of West Indies. J Dent Educ 2004;68:81-8.

18. Ryding HA, Murphy HJ. Assessing outcomes of curricular change: a view from program graduates. J Dent Educ 2001;65:422-6.

19. Widström E., Martinsson T, Nilsson B. Swedish and Finnish dental practitioners'opinions of their undergraduate education. Community Dent Oral Epidemiol 1988;16:139-42.
20. Diamanti N, Duxbury AJ, Ariyaratnam S, Macfarlane TV. Attitudes to biopsy procedures in general dental practice. Br Dent J 2002;192:588-92.

21. Burzynski NJ, Rankin KV, Silverman SJr, Scheetz JP, Jones DL. Graduating dental students' perceptions of oral cancer education: results of an exit survey of seven dental schools. J Cancer Educ 2002;17:83-4.

22. Seoane J, Warnakulasuriya S, Varela-Centelles P, Esparza G., Dios PD. Oral cancer: experiences and diagnostic abilities elicited by dentists in North-western Spain. Oral Dis 2006;12:487-492.

23. Afsharzand Z, Lim MV, Rashedi B, Petropoulos VC. Predoctoral implant dentistry curriculum survey: European dental schools. Eur J Dent Educ 2005;9:3745.

24. De Bruyn H, Koole S, Mattheos, Lang NP. A survey on undergraduate implant dentistry education in Europe. Eur J Dent Educ 2009;13:3-9

Correspondance et offprints : Valérie Roger-Leroi, UFR d'odontologie, 11 boulevard Charles de Gaulle, 63000 Clermont-Ferrand, France. Téléphone : + (0)4 731773 32, télécopie : + (0)4 73177309. Mailto : valerie.roger@u-clermont1.fr 